\title{
A TRAJECTORIAL PROOF OF THE VORTEX METHOD FOR THE TWO-DIMENSIONAL NAVIER-STOKES EQUATION
}

\author{
By SYLVIE MÉLÉARD \\ Université Paris 10
}

\begin{abstract}
We consider the Navier-Stokes equation in dimension 2 and more precisely the vortex equation satisfied by the curl of the velocity field. We show the relation between this equation and a nonlinear stochastic differential equation. Next we use this probabilistic interpretation to construct approximating interacting particle systems which satisfy a propagation of chaos property: the laws of the empirical measures tend, as the number of particles tends to $\infty$, to a deterministic law for which marginals are solutions of the vortex equation. This pathwise result justifies completely the vortex method introduced by Chorin to simulate the solutions of the vortex equation. Our approach is inspired by Marchioro and Pulvirenti and we improve their results in a pathwise sense.
\end{abstract}

1. Introduction. In this paper we consider the equation describing the velocity of an incompressible fluid in dimension 2 . This equation on the velocity field of the fluid is the well-known Navier-Stokes equation and in dimension 2, it can be expressed as an equation for the curl of the velocity. In this simplified form, the Navier-Stokes equation appears as a McKean-Vlasov equation, in which the coefficient of the drift term $K$ can explode.

About 20 years ago, Chorin proposed a vortex method to simulate the solutions of this equation. His method consisted of considering some cutoff drift kernels, assuming the convergence of the cutoff model to the original model and simulating some cutoff particle approximations. His approach was not mathematically proved and many authors tried to give a proof of the convergence.

In 1982, Marchioro and Pulvirenti gave a probabilistic interpretation of the Navier-Stokes equation due to a nonlinear diffusion. They introduced rigorously a cutoff model and some particle systems and proved a result of convergence for the expectations of the empirical measures of the particle systems at each fixed time to the solution at this time of the Navier-Stokes equation with bounded integrable initial data.

Then an open question was the pathwise convergence of these empirical measures to the law of the nonlinear diffusion, and as an equivalent result the propagation of chaos for the interacting particle systems.

In 1987, Osada proved a propagation of chaos result for an interacting particle system without cutoff by an analytical method based on generators of generalized divergence form, but only for large viscosities and for bounded

Received March 1999; revised January 2000.

AMS 1991 subject classifications. Primary 60K35; secondary 76D05.

Key words and phrases. Two-dimensional Navier-Stokes equation, vortex method, interacting particle systems, propagation of choas. 
density initial data. The tightness of the laws of the particle systems is always true and the constraint on the viscosity appears in the identification of the limit laws.

In this paper, we consider the framework introduced by Marchioro and Pulvirenti, and give a trajectorial proof of the vortex method by showing a propagation of chaos result for the stochastic interacting particle system. For each size $n$, the $n$-particle system is defined with a cutoff drift kernel with cutoff depending on $n$ in a precise asymptotics given by the estimates. One proves that the laws of the particle systems converge, and also the empirical measures, as probability measures on the path space, to a probability measure whose time marginals have density functions with respect to the Lebesgue measure, which are solutions of the Navier-Stokes equation. One obtains a trajectorial rate of convergence, which is unfortunately certainly not optimal.

As written at the end of [7], Marchioro and Pulvirenti wished to describe a unified approach for Navier-Stokes and Euler equations. So they did not fully exploit the stochastic nature of the Navier-Stokes equation, which we do in this paper. Our results, performed directly on the path space, yield the asymptotics of the path functionals such as hitting times or suprema over time intervals. The Euler equation cannot have the same type of stochastic interpretation since it lacks a Laplacian term, and the exact estimate we obtain in Corollary 3.6 explodes when the viscosity tends to 0. Then our approach cannot give better results on the Euler equation than the ones obtained in [7].

We consider here as initial data for the Navier-Stokes equation a bounded integrable function, not only a bounded density function. We are inspired by a paper of Jourdain [3], which shows how to take into account bounded signed measures instead of probability measures as initial conditions in a McKean-Vlasov context. One considers probability measures on the path space such that each sample path has a signed weight depending on the initial data. This trick allows us to have a simpler approach of the initial behavior than in Marchioro and Pulvirenti.

Notation. We will denote by $|\cdot|$ the Euclidean norm in $\mathbb{R}^{2}$, by $\|\cdot\|_{\infty}$ the $L^{\infty}$-norm and by $\|\cdot\|_{1}$ the $L^{1}$-norm in $\mathbb{R}^{2}$. The letter $C$ will denote a real constant which can change from line to line.

1.1. The vortex equation. Let us consider the velocity flow $v(t, x), t \in \mathbb{R}_{+}$, $x \in \mathbb{R}^{2}$, of a viscous and incompressible fluid in the whole plane. The governing equation of this motion is the Navier-Stokes equation given by

$$
\begin{aligned}
& \frac{\partial v}{\partial t}(t, x)+(v \cdot \nabla) v(t, x)=\nu \Delta v(v, x)-\nabla p, \\
& \nabla \cdot v(x, t)=0, \quad v(x, t) \rightarrow 0 \quad \text { as }|x| \rightarrow+\infty \text { for } 0 \leq t<+\infty,
\end{aligned}
$$

where $p$ is the pressure and $\nu>0$ the viscosity (assumed to be constant).

It is well known that the Navier-Stokes equation in dimension 2 can be reformulated in terms of the vorticity flow $w(t, x)=\operatorname{curl} v(t, x)$. More 
precisely, the flow $w$ is solution of the nonlinear partial differential equation

$$
\begin{gathered}
\frac{\partial w}{\partial t}(t, x)+(v \cdot \nabla) w(t, x)=\nu \Delta w(t, x), \\
v(t, x)=\int_{\mathbb{R}^{2}} K(x-y) w(t, y) d y
\end{gathered}
$$

for initial data $w_{0}$, where $K(x)$ is the kernel of Biot and Savart defined by

$$
\forall x=\left(x_{1}, x_{2}\right) \in \mathbb{R}^{2}, \quad K(x)=\frac{1}{2 \pi} \frac{1}{\left(x_{1}^{2}+x_{2}^{2}\right)}\left(-x_{2}, x_{1}\right) .
$$

Note that $\nabla \cdot K=0$, implying $\nabla \cdot v=0$.

The main difficulty of the kernel $K$ is the explosion at 0 . However, if $w \in$ $L^{\infty} \cap L^{1}\left(\mathbb{R}^{2}\right)$, (1.3) makes sense, as can be seen as follows.

LEMMA 1.1. The function $K$ is bounded at $\infty$ and integrable near 0 . Let us introduce

$$
K_{1}=\int_{B(0,1)}|K(y)| d y, \quad K_{\infty}=\sup _{y \in B(0,1)^{c}}|K(y)| .
$$

Then, for every function $g \in L^{1} \cap L^{\infty}$ and $x \in \mathbb{R}^{2}$,

$$
|K * g(x)| \leq\|g\|_{\infty} K_{1}+\|g\|_{1} K_{\infty} .
$$

The proof is very simple, and (1.5) is the key point to get an existence and uniqueness result for the solution of (1.2) if the initial data is assumed to be bounded and integrable.

Theorem 1.2. Let us consider $w_{0} \in L^{1} \cap L^{\infty}, \nu>0$ and $T>0$. Then:

(i) There exists a unique solution $t \rightarrow w_{t} \in L^{1} \cap L^{\infty}\left(\mathbb{R}^{2}\right)$, a solution of (1.2) in the weak sense, which means that, $\forall \phi \in C_{b}^{2}\left(\mathbb{R}^{2}\right)$,

$$
\begin{aligned}
\int_{\mathbb{R}^{2}} \phi(x) w_{t}(x) d x \\
=\int_{\mathbb{R}^{2}} \phi(x) w_{0}(x) d x+\nu \int_{0}^{t} \int_{\mathbb{R}^{2}} \Delta \phi(x) w_{s}(x) d x d s \\
\quad+\int_{0}^{t} \int_{\mathbb{R}^{2}}\left(K * w_{s}(x) \cdot \nabla \phi(x)\right) w_{s}(x) d x d s .
\end{aligned}
$$

Moreover,

$$
\sup _{t \in[0, T]}\left\|w_{t}\right\|_{\infty} \leq\left\|w_{0}\right\|_{\infty}, \quad\left\|w_{t}\right\|_{1} \leq\left\|w_{0}\right\|_{1} \quad \forall t \in[0, T] .
$$

(ii) If the initial function $w_{0}$ is nonnegative, then it is the same for each $w_{t}, t \geq 0$. 
(iii) The function $w$ is a.s. solution of the evolution equation

$$
\begin{aligned}
w(t, x)= & G_{t}^{\nu} * w_{0}(x) \\
& +\int_{0}^{t} \int_{\mathbb{R}^{2}} \nabla_{x} G_{t-s}^{\nu}(x-y) \cdot K * w_{s}(y) w_{s}(y) d y d s,
\end{aligned}
$$

where $G_{t}^{\nu}$ is the heat kernel in $\mathbb{R}^{2}$ defined by $G_{t}^{\nu}(x)=1 / 4 \pi t \nu e^{-\left(|x|^{2} / 4 t \nu\right)}$.

Proof. (i) The existence and uniqueness of the weak solution of (1.2) with initial data $w_{0}$ in $L^{1} \cap L^{\infty}$ are proved, for example, in Marchioro and Pulvirenti [7], as (1.7) and the positivity.

(ii) By using Fubini's theorem (allowed by Lemma 1.1 and applied to $w_{s}$ ), we can easily prove that, for every function $\psi(t, x) \in C_{b}^{1,2}\left(\mathbb{R}_{+} \times \mathbb{R}^{2}\right)$,

$$
\begin{aligned}
\int_{\mathbb{R}^{2}} \psi(t, x) w_{t}(x) d x & \\
= & \int_{\mathbb{R}^{2}} \psi(0, x) w_{0}(x) d x+\nu \int_{0}^{t} \int_{\mathbb{R}^{2}} \Delta \psi(s, x) w_{s}(x) d x d s \\
& \quad+\int_{0}^{t} \int_{\mathbb{R}^{2}} \psi_{s}^{\prime}(s, x) w_{s}(x) d x d s+\int_{0}^{t} \int_{\mathbb{R}^{2}}\left(K * w_{s}(x) \cdot \nabla_{x} \psi(s, x)\right) w_{s}(x) d x d s .
\end{aligned}
$$

Then by choosing for a fixed time $t, \psi(s, x)=\int_{\mathbb{R}^{2}} G_{t-s}^{\nu}(x-y) \phi(y) d y$ for $\phi \in C_{b}^{2}\left(\mathbb{R}^{2}\right)$, we obtain the evolution equation (1.8). In fact, a direct proof of the existence and uniqueness of the solution of (1.2) in $L^{1} \cap L^{\infty}$ can be given by studying (1.8) (see Giga, Miyakawa and Osada [2]).

Let us remark that a simple computation gives an estimate of the $L^{1}$-norm of the gradient of $G_{t}^{\nu}$.

LEMMA 1.3.

$$
\left\|\nabla_{x} G_{t}^{\nu}\right\|_{L^{1}\left(\mathbb{R}^{2}\right)} \leq \frac{A}{\sqrt{\nu t}}
$$

where $A$ is a real constant and then $\int_{0}^{t}\left\|\nabla_{x} G_{t-s}^{\nu}\right\|_{L^{1}\left(\mathbb{R}^{2}\right)} d s<+\infty$.

2. The nonlinear martingale problem and the stochastic differential equation associated with the two-dimensional Navier-Stokes equation. We are in a McKean-Vlasov context. If the initial function $w_{0}$ is a density function on $\mathbb{R}^{2}$, it is natural to associate with the weak form (1.6) of the Navier-Stokes equation a nonlinear martingale problem and a nonlinear stochastic differential equation, by interpreting a solution as the timemarginal flow of a probability measure on $C\left([0, T], \mathbb{R}^{2}\right)$. Here, we will use a trick due to Jourdain [4] to pass from a density function to every bounded integrable function.

In the following discussion, we will consider an initial bounded and integrable function $w_{0}$ on $\mathbb{R}^{2}$. We assume moreover that $w_{0}$ is not the function identically equal to 0 . 
We define the bounded function $h$ from the initial condition $w_{0}$ by

(2.1) $\forall x \in \mathbb{R}^{2}, \quad h(x)=\frac{w_{0}(x)\left\|w_{0}\right\|_{1}}{\left|w_{0}(x)\right|}, \quad$ with the convention $\frac{0}{0}=0$.

Let us remark that, for each $x \in \mathbb{R}^{2}$,

$$
-\left\|w_{0}\right\|_{1} \leq h(x) \leq\left\|w_{0}\right\|_{1}
$$

and that

$$
w_{0}(x) d x=h(x) \frac{\left|w_{0}(x)\right|}{\left\|w_{0}\right\|_{1}} d x, \quad \text { where } \frac{\left|w_{0}\right|}{\left\|w_{0}\right\|_{1}},
$$

is thus a density of probability measure.

Now, for $Q$ a probability measure on $C\left([0,+\infty], \mathbb{R}^{2}\right)$, we define the flow $\left(\widetilde{Q}_{t}\right)_{t \geq 0}$ of signed measures on $\mathbb{R}^{2}$ by

$$
\forall B \text { Borel subset of } \mathbb{R}^{2}, \quad \widetilde{Q}_{t}(B)=E^{Q}\left(1_{B}\left(X_{t}\right) h\left(X_{0}\right)\right),
$$

where $X$ denotes the canonical process on $C\left([0,+\infty), \mathbb{R}^{2}\right)$. (One associates with each sample path a signed weight depending on the initial position.)

Lemma 2.1 will be useful in the following discussion.

LEMMA 2.1. (i) For each $t \geq 0$, the signed measure $\widetilde{Q}_{t}$ is bounded, and its total mass is less than $\left\|w_{0}\right\|_{1}$.

(ii) If $Q_{t}$ is absolutely continuous with respect to the Lebesgue measure, then $\widetilde{Q}_{t}$ also has a bounded density.

PRoof. Since the function $h$ is bounded by $\left\|w_{0}\right\|_{1}$ and using (2.3), the lemma is obvious.

Equation (1.2) understood in its weak form leads naturally to the following definition.

DEFINITION 2.2. The probability measure $P \in \mathscr{P}\left(C\left(\mathbb{R}_{+}, \mathbb{R}^{2}\right)\right)$ is a solution of the nonlinear martingale problem $(\mathscr{M})$ if, for each $\phi \in C_{b}^{2}\left(\mathbb{R}^{2}\right)$,

$$
\phi\left(X_{t}\right)-\phi\left(X_{0}\right)-\int_{0}^{t} K * \widetilde{P}_{s}\left(X_{s}\right) \cdot \nabla \phi\left(X_{s}\right) d s-\nu \int_{0}^{t} \Delta \phi\left(X_{s}\right) d s
$$

is a $P$-martingale, where $X$ is the canonical process on $C\left(\mathbb{R}_{+}, \mathbb{R}^{2}\right), P_{0}$ has the law $\left(\left|w_{0}(x)\right| /\left\|w_{0}\right\|_{1}\right) d x$ and $P_{s}=P \circ X_{s}^{-1}$.

This nonlinear martingale problem is related to the following nonlinear stochastic differential equation. 
DEFINITION 2.3. Let us consider a random $\mathbb{R}^{2}$-valued variable $X_{0}$ with distribution $\left(\left|w_{0}(x)\right| /\left\|w_{0}\right\|_{1}\right) d x$, and let $B$ be a two-dimensional Brownian motion independent of $X_{0}$. A solution $X \in C\left(\mathbb{R}_{+}, \mathbb{R}^{2}\right)$ of the nonlinear stochastic differential equation satisfies, $\forall t \in \mathbb{R}_{+}$,

$$
\begin{aligned}
& X_{t}=X_{0}+\sqrt{2 \nu} B_{t}+\int_{0}^{t} K * \widetilde{P}_{s}\left(X_{s}\right) d s, \\
& P_{s} \text { is the marginal at time } s \text { of the law of } X_{s} .
\end{aligned}
$$

Notation. We denote by $\widehat{\mathscr{P}}_{\infty}\left(C\left(\mathbb{R}_{+}, \mathbb{R}^{2}\right)\right)$ the space of probability measures on $C\left(\mathbb{R}_{+}, \mathbb{R}^{2}\right)$ whose marginals $P_{s}$ at time $s$ are absolutely continuous with respect to the Lebesgue measure on $\mathbb{R}^{2}$, for every $s \in \mathbb{R}_{+}$, and have a density in $L^{\infty}\left(\mathbb{R}^{2}\right)$. For such probability measures, there exists a measurable function $(s, x) \rightarrow \tilde{p}(s, x)$ in $L^{\infty}$ such that, $\forall s \in[0, T], \widetilde{P}_{s}(d x)=\tilde{p}(s, x) d x$ (cf. Meyer [9], page 194). We call this function $\tilde{p}$ a measurable version of the densities of $\left(P_{s}\right)$. We will prove the following theorem.

THEOREM 2.4. Let us consider a bounded integrable function $w_{0}$. Then there exists a unique solution $P \in \widehat{\mathscr{P}}_{\infty}\left(C\left(\mathbb{R}_{+}, \mathbb{R}^{2}\right)\right)$ to the martingale problem $(\mathscr{M})$ such that $P_{0}(d x)=\left(\left|w_{0}(x)\right| /\left\|w_{0}\right\|_{1}\right) d x$.

Moreover, for each $t \in \mathbb{R}_{+}$, the signed bounded measure $\widetilde{P}_{t}$ is absolutely continuous with respect to the Lebesgue measure, and each measurable version of its density is almost surely bounded and equal to the function $w_{t}$ defined in Theorem 1.2.

Proof. (i) If $P \in \widehat{\mathscr{P}}_{\infty}\left(C\left([0, T], \mathbb{R}^{2}\right)\right)$ is a solution of $(\mathscr{M})$ and $\tilde{p}_{t}$ a measurable version of the densities of $\widetilde{P}_{t}$, then by multiplying by $h\left(X_{0}\right)$ and by taking the expectation in $(\mathscr{M})$, we get that $\tilde{p}$ is a weak solution of (1.2) with initial condition $w_{0}$. Then by the uniqueness given in Theorem 1.2, for each $t \in \mathbb{R}_{+}, \tilde{p}_{t}(x)=w_{t}(x)$ a.s.

(ii) Let us consider this unique weak solution $w$ of (1.2) issued from $w_{0}$. Then $w_{t}$ is for each $t$ a bounded integrable function. We say that $P^{w} \in$ $\mathscr{P}\left(C\left(\mathbb{R}_{+}, \mathbb{R}^{2}\right)\right)$ is a solution of the classical martingale problem $\left(\mathscr{M}^{w}\right)$ if, for each $\phi \in C_{b}^{2}\left(\mathbb{R}^{2}\right)$,

$$
\phi\left(X_{t}\right)-\phi\left(X_{0}\right)-\nu \int_{0}^{t} \Delta \phi\left(X_{s}\right) d s-\int_{0}^{t} K * w_{s}\left(X_{s}\right) \cdot \nabla \phi\left(X_{s}\right) d s
$$

is a $P^{w}$-martingale and $P_{0}^{w}(d x)=\left(\left|w_{0}(x)\right| /\left\|w_{0}\right\|_{1}\right) d x$. This martingale problem is well posed. Indeed, by Lemma 1.1 and (1.7), for each $s \geq 0$,

$$
\left\|K * w_{s}\right\|_{\infty} \leq K_{1}\left\|w_{s}\right\|_{\infty}+K_{\infty}\left\|w_{s}\right\|_{1} \leq K_{1}\left\|w_{0}\right\|_{\infty}+K_{\infty}\left\|w_{0}\right\|_{1}=\widehat{K}\left(w_{0}\right)
$$

so the drift coefficient is bounded, and by Girsanov's theorem, we get the existence and uniqueness of the solution of $\left(\mathscr{M}^{w}\right)$. Moreover, every time marginal of $P^{w}$ admits a density $P_{s}^{w}$, and multiplying by $h\left(X_{0}\right)$ all the terms of the 
martingale problem and taking expectations, we obtain immediately that the flow $\left(\tilde{p}_{s}^{w}\right)_{s \in \mathbb{R}_{+}}$is a solution of the weak equation: for each $\phi \in C_{b}^{2}\left(\mathbb{R}^{2}\right)$,

$$
\begin{aligned}
\int_{\mathbb{R}^{2}} \phi(x) \tilde{p}_{t}^{w}(x) d x & \\
= & \int_{\mathbb{R}^{2}} \phi(x) w_{0}(x) d x+\nu \int_{0}^{t} \int_{\mathbb{R}^{2}} \Delta \phi(x) \tilde{p}_{s}^{w}(x) d x d s . \\
& +\int_{0}^{t} \int_{\mathbb{R}^{2}}\left(K * w_{s}(x) \cdot \nabla \phi(x) \tilde{p}_{s}^{w}(x) d x d s\right) .
\end{aligned}
$$

Then the flow $\tilde{p}_{t}$ is a solution of

$$
\frac{\partial \tilde{p}^{w}}{\partial t}+(K * w \cdot \nabla) \tilde{p}^{w}=\nu \triangle \tilde{p}^{w}, \quad \tilde{p}_{0}^{w}=w_{0} .
$$

Since the divergence of the drift term is equal to 0 , we can adapt the proof of Theorem 6.2.2 in Lewandowski [6] and obtain, for each $t \in \mathbb{R}_{+}$, for each $q \in \mathbb{R}_{+}^{*}$,

$$
\left\|\tilde{p}_{t}^{w}\right\|_{q} \leq\left\|w_{0}\right\|_{q},
$$

and since $w_{0}$ belongs to $L^{\infty} \cap L^{1}$, we conclude by taking $q \rightarrow+\infty$ that, for each $t \in[0, T]$,

$$
\left\|\tilde{p}_{t}^{w}\right\|_{\infty} \leq\left\|w_{0}\right\|_{\infty}
$$

(iii) Let us now prove the existence and uniqueness of a solution of (2.6) in $L^{\infty}\left([0, T], L^{1} \cap L^{\infty}\right)$. We obtain as before by Fubini's theorem and due to Lemma 1.1 that $\tilde{p}^{w}$ is a solution of the following evolution equation:

$$
\begin{aligned}
\tilde{p}^{w}(t, x)= & G_{t}^{\nu} * w_{0}(x) \\
& +\int_{0}^{t} \int \nabla_{x} G_{t-s}^{\nu}(x-y) K * w_{s}(y) \tilde{p}^{w}(s, y) d y .
\end{aligned}
$$

Let us prove the uniqueness of the solution of $(2.8)$ in $L^{\infty}\left([0, T], L^{\infty}\right)$. Let $q$ and $q^{\prime}$ be two solutions. Then

$$
\begin{aligned}
& q(t, x)-q^{\prime}(t, x) \\
& \quad=\int_{0}^{t} \int \nabla_{x} G_{t-s}^{\nu}(x-y) K * w_{s}(y)\left(q(s, y)-q^{\prime}(s, y)\right) d y d s .
\end{aligned}
$$

Thus, by (1.9) and (2.5),

$$
\begin{aligned}
& \left\|q(t, \cdot)-q^{\prime}(t, \cdot)\right\|_{\infty} \\
& \quad \leq \int_{0}^{t}\left\|\int \nabla_{x} G_{t-s}^{\nu}(x-y) K * w_{s}(y)\left(q(s, y)-q^{\prime}(s, y)\right) d y\right\|_{\infty} d s \\
& \quad \leq \widehat{K}\left(w_{0}\right) \int_{0}^{t}\left\|q(s, \cdot)-q^{\prime}(s, \cdot)\right\|_{\infty}\left\|\nabla_{x} G_{t-s}^{\nu}\right\|_{\infty} d s \\
& \quad \leq \frac{A \widehat{K}\left(w_{0}\right)}{\sqrt{\nu}} \int_{0}^{t} \frac{\left\|q(s, \cdot)-q^{\prime}(s, \cdot)\right\|_{\infty}}{\sqrt{t-s}} d s .
\end{aligned}
$$


We are almost in a standard Gronwall situation. By an iteration, we obtain

$$
\begin{aligned}
\| q( & (, \cdot)-q^{\prime}(t, \cdot) \|_{\infty} \\
& \leq C \int_{0}^{t} \frac{1}{\sqrt{t-s}} \int_{0}^{s} \frac{\left\|q(u, \cdot)-q^{\prime}(u, \cdot)\right\|_{\infty}}{\sqrt{s-u}} d u d s \\
& \leq C \int_{0}^{t}\left\|q(u, \cdot)-q^{\prime}(u, \cdot)\right\|_{\infty} \int_{u}^{t} \frac{1}{\sqrt{t-s} \sqrt{s-u}} d s d u \\
& \leq C \int_{0}^{t}\left\|q(u, \cdot)-q^{\prime}(u, \cdot)\right\|_{\infty} d u
\end{aligned}
$$

Therefore,

$$
\sup _{t \in[0, T]}\left\|q(t, \cdot)-q^{\prime}(t, \cdot)\right\|_{\infty}=0
$$

and the uniqueness in (2.8) is proved. Hence $\tilde{p}^{w}$ is the unique solution of (2.8) and since $w$ satisfies this equation,

$$
\sup _{t \in[0, T]}\left\|\tilde{p}^{w}(t, \cdot)-w(t, \cdot)\right\|_{\infty}=0
$$

So the probability measure $P^{w}$ is a solution of the nonlinear martingale problem $(\mathscr{M})$.

(iv) Let us now prove the uniqueness of a solution of this martingale problem. Let $P$ and $Q$ be two solutions. By the same reasoning as before, it is easy to prove that $\left(\widetilde{P}_{t}\right)_{t>0}$ and $\left(\widetilde{Q}_{t}\right)_{t \geq 0}$ are equal to $(w(t, x) d x)_{t \geq 0}$. Hence $P$ and $Q$ are solutions of the classical well-posed martingale problem $\left(\mathscr{M}^{w}\right)$ and are then equal, and Theorem 2.4 is proved.

REMARK 2.5. The function $K * w$ is bounded but not Lipschitz. So we have existence and uniqueness in law for the nonlinear SDE (2.4), but we do not have a strong uniqueness result.

\section{An approximating system.}

3.1. A model with cutoff. Let us consider as in Marchioro and Pulvirenti [7] a cutoff kernel $K_{\varepsilon}$ defined in the following way. We denote by $g(r)=$ $-(1 / 2 \pi) \ln r$ the fundamental solution of the Poisson equation. One knows that, for $x \in \mathbb{R}^{2}, K(x)=\nabla^{\perp} g(|x|)$. For each $\varepsilon>0$, we consider $g_{\varepsilon}$ defined as $g_{\varepsilon}(r)=g(r)$ if $|r| \geq \varepsilon$ and arbitrarily extended to an even $C^{2}(\mathbb{R})$ function such that $\left|g_{\varepsilon}^{\prime}(r)\right| \leq\left|g^{\prime}(r)\right|$ and $\left|g_{\varepsilon}^{\prime \prime}(r)\right| \leq\left|g^{\prime \prime}(r)\right|$. Then we define

$$
K_{\varepsilon}(x)=\nabla^{\perp} g_{\varepsilon}(|x|) .
$$

The function $K_{\varepsilon}$ is then Lipschitz continuous and bounded and we denote by $M_{\varepsilon}$ the maximum value of $K_{\varepsilon}$ on $\mathbb{R}^{2}$ and by $L_{\varepsilon}$ a Lipschitz constant.

We now define the interacting particle system we are interested in. 
Definition 3.1. Consider a sequence $\left(B^{i}\right)_{i \in \mathbb{N}}$ of independent Brownian motions on $\mathbb{R}^{2}$ and an $\mathbb{R}^{2}$-valued sequence of independent variables $\left(Z_{0}^{i}\right)_{i \in \mathbb{N}}$ distributed according to $\left(\left|w_{0}(x)\right| /\left\|w_{0}\right\|_{1}\right) d x$ and independent of $\left(B^{i}\right)_{i \in \mathbb{N}}$. For a fixed $\varepsilon$, for each $n \in \mathbb{N}^{*}$ and $1 \leq i \leq n$, let us consider the interacting processes defined by

$$
Z_{t}^{i n, \varepsilon}=Z_{0}^{i}+\sqrt{2 \nu} B_{t}^{i}+\int_{0}^{t} K_{\varepsilon} * \tilde{\mu}_{s}^{n, \varepsilon}\left(Z_{s}^{i n, \varepsilon}\right) d s
$$

where

$$
\mu^{n, \varepsilon}=\frac{1}{n} \sum_{j=1}^{n} \delta_{Z^{j n, \varepsilon}} \in \mathscr{P}\left(C\left(\mathbb{R}_{+}, \mathbb{R}^{2}\right)\right)
$$

is the empirical measure of the system:

$$
\tilde{\mu}_{s}^{n, \varepsilon}=\frac{1}{n} \sum_{j=1}^{n} h\left(Z_{0}^{j}\right) \delta_{Z_{s}^{j n, \varepsilon} .}
$$

We also define the limiting independent processes by

$$
\bar{Z}_{t}^{i, \varepsilon}=Z_{0}^{i}+\sqrt{2 \nu} B_{t}^{i}+\int_{0}^{t} K_{\varepsilon} * \widetilde{P}_{s}^{\varepsilon}\left(\bar{Z}_{s}^{i, \varepsilon}\right) d s,
$$

where $P_{s}^{\varepsilon}$ is the law of $\bar{Z}_{s}^{i, \varepsilon}$.

Proposition 3.2. (i) For each $T>0$ and for each $n$, there exist a unique (pathwise) solution to the interacting particle system (3.1) in $C\left([0, T], \mathbb{R}^{2 n}\right)$ and a unique (pathwise) solution to the nonlinear equation (3.2) in $C([0$, $\left.T], \mathbb{R}^{2}\right)$.

(ii) For each $T>0$,

$$
E\left(\sup _{t \leq T}\left|Z_{t}^{i n, \varepsilon}-\bar{Z}_{t}^{i, \varepsilon}\right|\right) \leq \frac{M_{\varepsilon}}{\sqrt{n} L_{\varepsilon}} \exp \left(\left\|w_{0}\right\|_{1} T L_{\varepsilon}\right) .
$$

PRoOF. By the boundedness of $h$, the proof of the first assertion is standard and can be adapted from Sznitman [12], Theorem 1.1, and the second assertion comes from an easy adaptation of the computations in Jourdain and Méléard [4], Proposition 2.3. See also Jourdain [3].

3.2. The approximating interacting particle system. We now consider $T>0$ and a sequence $\left(\varepsilon_{n}\right)$ tending to 0 such that

$$
\lim _{n} \frac{M_{\varepsilon_{n}}}{\sqrt{n} L_{\varepsilon_{n}}} \exp \left(\left\|w_{0}\right\|_{1} T L_{\varepsilon_{n}}\right)=0
$$

with the notation of the previous section. For each $n$ and given independent Brownian motions $\left(B^{i}\right)_{1 \leq i \leq n}$, we consider a coupling between the $n$ particle system $\left(Z^{i n}=Z^{i n, \varepsilon_{n}}\right)$ defined with the drift $K_{\varepsilon_{n}}$ as in (3.1), and the 
corresponding $n$ independent limiting processes $\bar{Y}^{i n}=\bar{Z}^{i, \varepsilon_{n}}$ defined for each $t \leq T$ and $n$ by

$$
\bar{Y}_{t}^{i n}=Z_{0}^{i}+\sqrt{2 \nu} B_{t}^{i}+\int_{0}^{t} K_{\varepsilon_{n}} * \widetilde{P}_{s}^{n}\left(\bar{Y}_{s}^{i n}\right) d s,
$$

where $P_{s}^{n}$ is the common law of $\bar{Y}_{s}^{i n}$.

By the same arguments as before, $P_{s}^{n}$ admits a density function $p_{s}^{n}$ and then $\widetilde{P}_{s}^{n}$ admits a density function $\tilde{p}_{s}^{n}$, which is a weak solution of the equation

$$
\frac{\partial \tilde{p}^{n}}{\partial t}=\nu \Delta \tilde{p}^{n}-\left(K_{\varepsilon_{n}} * \tilde{p}^{n} \cdot \nabla\right) \tilde{p}^{n}, \quad p_{0}^{n}=w_{0} .
$$

Indeed, to prove it, we compute $\phi\left(\bar{Y}_{t}^{i n}\right)$ for a smooth function $\phi$ by Itô's formula, multiply it by $h\left(Z_{0}^{i}\right)$ and take expectations. By the same reasoning as in the proof of Theorem 2.4 and since $w_{0} \in L^{1} \cap L^{\infty}$ and $K_{\varepsilon_{n}} * \tilde{p}^{n}$ is bounded by $M_{\varepsilon_{n}}$ and has a divergence equal to 0 , we can prove the following result.

PROPOSITION 3.3.

$$
\sup _{n} \sup _{t \in[0, T] ; x \in \mathbb{R}^{2}}\left|\tilde{p}_{t}^{n}(x)\right| \leq\left\|w_{0}\right\|_{\infty} .
$$

It is moreover easy to prove by standard arguments that $\tilde{p}^{n}$ is a solution of the evolution equation

$$
\tilde{p}_{t}^{n}(x)=G_{t}^{\nu} * w_{0}(x)+\int_{0}^{t} \nabla_{x} G_{t-s}^{\nu} *\left(\tilde{p}_{s}^{n} \cdot K_{\varepsilon_{n}} * \tilde{p}_{s}^{n}\right)(x) d s .
$$

Let us now introduce for each $n$ the coupling of processes $\left(Z^{i n}, \bar{Y}^{i n}\right.$, $\left.\bar{X}^{i}\right)_{1 \leq i \leq n}$, where $\left(\bar{X}^{i}\right)$ are independent copies of $X$ defined as in (2.4) on a certain probability space and $Z^{i n}, \bar{Y}^{i n}$ are driven, for each $i$ respectively, following the same Brownian motion as $\bar{X}^{i}$. We will now compare the two processes $\bar{Y}^{i n}$ and $\bar{X}^{i}$. So, we need to estimate $w-\tilde{p}^{n}$. Using (1.8) and (3.8), we obtain

$$
\begin{aligned}
\tilde{p}_{t}^{n}(x)-w_{t}(x)= & \int_{0}^{t} \int_{\mathbb{R}^{2}} \nabla_{x} G_{t-s}^{\nu}(x-y) \\
& \times\left(K_{\varepsilon^{n}} * \tilde{p}_{s}^{n}(y) \tilde{p}_{s}^{n}(y)-K * w_{s}(y) w_{s}(y)\right) d y d s .
\end{aligned}
$$

We will prove the following result.

THEOREM 3.4. Let us define the norm

$$
\|\mid\| f\|=\| f\left\|_{1}+\right\| f \|_{\infty} .
$$

The space of real functions $f$ on $\mathbb{R}^{2}$ such that $\||f|\|<+\infty$ is obviously a complete space. Then

$$
\sup _{t \leq T}||\left|\tilde{p}_{t}^{n}-w_{t}\right| \| \leq\left.\frac{C_{1}}{\sqrt{\nu}} \varepsilon_{n}||\left|w_{0}\right|\right|^{2} \sqrt{T} \exp \left(C_{2}||\left|w_{0}\right||| T\right),
$$

where $C_{1}$ and $C_{2}$ are real constants. 
The proof begins with a lemma.

LEMMA 3.5. For each $t \leq T$, for each $x \in \mathbb{R}^{2}$,

$$
\left|\int_{\mathbb{R}^{2}}\left(K_{\varepsilon_{n}}(x-y)-K(x-y)\right) \tilde{P}_{t}^{n}(y) d y\right| \leq 2 \varepsilon_{n}\left\|w_{0}\right\|_{\infty} .
$$

and then

$$
\left\|K_{\varepsilon_{n}} * \tilde{p}_{t}^{n}-K * w_{t}\right\|_{\infty} \leq 2 \varepsilon_{n}\left\|w_{0}\right\|_{\infty}+\left(K_{1}+K_{\infty}\right) \mid\left\|\tilde{p}_{t}^{n}-w_{t}\right\| \| .
$$

Proof. (i) Since $K_{\varepsilon_{n}}$ and $K$ coincide for $|x| \geq \varepsilon_{n}$, we have

$$
\begin{aligned}
& \int_{\mathbb{R}^{2}}\left(K_{\varepsilon_{n}}(x-y)-K(x-y)\right) \tilde{p}_{t}^{n}(y) d y \\
& \quad \leq \int_{|x-y| \leq \varepsilon_{n}}\left|K_{\varepsilon_{n}}(x-y)-K(x-y)\right|\left|\tilde{p}_{t}^{n}(y)\right| d y \\
& \quad \leq \int_{|x-y| \leq \varepsilon_{n}}\left(\left|K_{\varepsilon_{n}}(x-y)\right|+|K(x-y)|\right)\left|\tilde{p}_{t}^{n}(y)\right| d y \\
& \quad \leq 2 \int_{|x-y| \leq \varepsilon_{n}}|K(x-y)|\left|\tilde{p}_{t}^{n}(y)\right| d y \\
& \quad \leq 2\left\|w_{0}\right\|_{\infty} \int_{|z| \leq \varepsilon_{n}}|K(z)| d z \quad \text { by (3.7) } \\
& \quad \leq 2 \varepsilon_{n}\left\|w_{0}\right\|_{\infty} \quad \text { by an easy computation. }
\end{aligned}
$$

We have used that, by definition, $\left|K_{\varepsilon_{n}}(x-y)\right| \leq|K(x-y)|$.

(ii) For $x \in \mathbb{R}^{2}$,

$$
\begin{aligned}
& \left|K_{\varepsilon_{n}} * \tilde{p}_{t}^{n}(x)-K * w_{t}(x)\right| \\
& \quad \leq 2 \varepsilon_{n}\left\|w_{0}\right\|_{\infty}+\int_{\mathbb{R}^{2}}|K(x-y)|\left|\tilde{p}_{t}^{n}(y)-w_{t}(y)\right| d y \\
& \quad \leq 2 \varepsilon_{n}\left\|w_{0}\right\|_{\infty}+K_{\infty}\left\|\tilde{p}_{t}^{n}-w_{t}\right\|_{1}+K_{1}\left\|\tilde{p}_{t}^{n}-w_{t}\right\|_{\infty} .
\end{aligned}
$$

Let us now prove Theorem 3.4.

Proof. We consider (3.9). Then

$$
\begin{aligned}
& \left|\tilde{p}_{t}^{n}(x)-w_{t}(x)\right| \\
& \leq \mid \int_{0}^{t} \int_{\mathbb{R}^{2}} \nabla_{x} G_{t-s}^{\nu}(x-y) \cdot\left(\tilde{p}_{s}^{n}(y)\left(K_{\varepsilon_{n}} * \tilde{p}_{s}^{n}(y)-K * w_{s}(y)\right)\right. \\
& \left.+K * w_{s}(y)\left(\tilde{p}_{s}^{n}(y)-w_{s}(y)\right)\right) d y d s \mid
\end{aligned}
$$




$$
\begin{aligned}
\leq \int_{0}^{t} \int_{\mathbb{R}^{2}}\left|\nabla_{x} G_{t-s}^{\nu}(x-y)\right|\left(| \tilde { p } _ { s } ^ { n } ( y ) | \left(2 \varepsilon_{n}\left\|w_{0}\right\|_{\infty}+(\right.\right. & \left.\left.K_{\infty}+K_{1}\right)||\left|\tilde{p}_{s}^{n}-w_{s}\right|||\right) \\
& \left.+\widehat{K}\left(w_{0}\right)||\left|\tilde{p}_{s}^{n}-w_{s}\right|||\right) d y d s \mid
\end{aligned}
$$

$$
\begin{aligned}
& \leq \frac{A}{\sqrt{\nu}} \int_{0}^{t} \frac{1}{\sqrt{t-s}}\left(\left\|w_{0}\right\|_{\infty}\left(2 \varepsilon_{n}\left\|w_{0}\right\|_{\infty}+\left(K_{\infty}+K_{1}\right)\left|\left\|\tilde{p}_{s}^{n}-w_{s}\right\|\right|\right)\right. \\
& \left.+\widehat{K}\left(w_{0}\right)\left|\left\|\tilde{p}_{s}^{n}-w_{s}\right\|\right|\right) d s \\
& \leq \frac{A}{\sqrt{\nu}}\left(4 \varepsilon_{n}\left\|w_{0}\right\|_{\infty}^{2} \sqrt{T}+2\left(K_{\infty}+K_{1}\right)||\left|w_{0}\right|\left\|\int_{0}^{t} \frac{1}{\sqrt{t-s}}\left|\left\|\tilde{p}_{s}^{n}-w_{s}\right\|\right| \mid d s\right) .\right.
\end{aligned}
$$

Consider now the $L^{1}$-norm of $\tilde{p}_{t}^{n}-w_{t}$ and in a similar way,

$$
\begin{aligned}
\int_{\mathbb{R}^{2}}\left|\tilde{p}_{t}^{n}(x)-w_{t}(x)\right| d x \\
\leq \int_{0}^{t} \frac{1}{\sqrt{t-s}}\left\|\tilde{p}_{s}^{n}\right\|_{1}\left(2 \varepsilon_{n}\left\|w_{0}\right\|_{\infty}+\left(K_{\infty}+K_{1}\right)\left\|\left|\tilde{p}_{s}^{n}-w_{s} \|\right|\right) d s\right. \\
\quad+\int_{0}^{t} \frac{1}{\sqrt{t-s}}\left\|K * w_{s}\right\|_{\infty}\left\|\tilde{p}_{s}^{n}-w_{s}\right\|_{1} d s \\
\quad \leq \frac{A}{\sqrt{\nu}}\left(4 \varepsilon_{n}\left|\left\|w_{0}\right\|\right|^{2} \sqrt{T}+2\left(K_{\infty}+K_{1}\right)\left|\left\|w_{0}\left|\left\|\int_{0}^{t} \frac{1}{\sqrt{t-s}}\right\| \tilde{p}_{s}^{n}-w_{s}\right|\right\| d s\right) .\right.
\end{aligned}
$$

By associating the two previous results, we obtain

$$
||\left|\tilde{p}_{t}^{n}-w_{t}\right| \| \leq \frac{A}{\sqrt{\nu}}\left(8 \varepsilon_{n}||\left|w_{0}\right|||^{2} \sqrt{T}+2||\left|w_{0}\right||| \int_{0}^{t} \frac{1}{\sqrt{t-s}}||\left|\tilde{p}_{s}^{n}-w_{s}\right||| d s\right) .
$$

We iterate twice this inequality and obtain finally by Gronwall's lemma that

$$
\sup _{t \leq T}||\left|\tilde{p}_{t}^{n}-w_{t}\right||| \leq\left.\frac{C_{1}}{\sqrt{\nu}} \varepsilon_{n}||\left|w_{0}\right|\right|^{2} \sqrt{T} \exp \left(C_{2}||\left|w_{0}\right| \mid T\right)
$$

Adding now (3.12) and (3.10), we deduce the following result.

COROLlaRY 3.6. For each $t \leq T$,

$$
\left\|K_{\varepsilon_{n}} * \tilde{p}_{t}^{n}-K * w_{t}\right\|_{\infty} \leq A_{T} \varepsilon_{n}
$$

where

$$
A_{T}=2\left\|w_{0}\right\|_{\infty}+\left.\left(K_{\infty}+K_{1}\right) \frac{C_{1}}{\sqrt{\nu}} \varepsilon_{n}||\left|w_{0}\right|\right|^{2} \sqrt{T} \exp \left(C_{2}\left|\left\|w_{0}\right\|\right| T\right) .
$$

We are now able to obtain our main theorem. 
THEOREM 3.7. Let us consider a sequence of limiting independent processes solution on a certain probability space of the stochastic differential equation

$$
\bar{X}_{t}^{i}=Z_{0}^{i}+\sqrt{2 \nu} B_{t}^{i}+\int_{0}^{t} K * \tilde{p}_{s}\left(\bar{X}_{s}^{i}\right) d s,
$$

where $\left(B^{i}\right)_{i \in \mathbb{N}}$ are independent Brownian motions on $\mathbb{R}^{2}$ and $\left(Z_{0}^{i}\right)_{i \in \mathbb{N}}$ are $\mathbb{R}^{2}$. valued iid random variables independent of $\left(B^{i}\right)_{i \in \mathbb{N}}$ and with law $P_{0}(d x)=$ $\left(\left|w_{0}(x)\right| /\left\|w_{0}\right\|_{1}\right) d x, w_{0}$ being a bounded integrable function on $\mathbb{R}^{2}$. The probability measure $P_{s}$ is the common law of the $\bar{X}_{s}^{i}$, and $\tilde{p}_{s}$ the density of the signed measure $\widetilde{P}_{s}$ associated with $P_{s}$ by (2.3).

On the other hand, let us consider $T>0$ and a sequence $\left(\varepsilon_{n}\right)_{n \in \mathbb{N}}$ of real numbers such that (with the notation of Section 3.1),

$$
\lim _{n} \frac{M_{\varepsilon_{n}}}{\sqrt{n} L_{\varepsilon_{n}}} \exp \left(\left\|w_{0}\right\|_{1} T L_{\varepsilon_{n}}\right)=0 .
$$

We consider on the same probability space the coupled n-particle system $\left(Z^{\text {in }}\right)_{1 \leq i \leq n}$ defined by

$$
Z_{t}^{i n}=Z_{0}^{i}+\sqrt{2 \nu} B_{t}^{i}+\int_{0}^{t} \frac{1}{n} \sum_{j=1}^{n} h\left(Z_{0}^{j}\right) K_{\varepsilon_{n}}\left(Z_{s}^{i n}-Z_{s}^{j n}\right) d s .
$$

Then, for each $1 \leq i \leq n$,

$$
\lim _{n \rightarrow+\infty} E\left(\sup _{t \leq T}\left|Z_{t}^{i n}-\bar{X}_{t}^{i}\right|\right)=0
$$

[in the precise asymptotics given by (3.20)].

This implies the propagation of chaos and the convergence in law (uniformly in time) of the weighted empirical measures $\tilde{\mu}_{s}^{n}=(1 / n) \sum_{i=1}^{n} h\left(Z_{0}^{i}\right) \delta_{Z_{s}^{i n}}$ to $\widetilde{P}_{s}$ associated with the law of $\bar{X}^{i}$, and $\widetilde{P}_{s}$ has a density equal to the solution $w$ of the Navier-Stokes equation with initial data $w_{0}$, as seen in Theorem 2.4.

Proof.

$$
\begin{aligned}
E\left(\sup _{t \leq T}\left|Z_{t}^{i n}-\bar{X}_{t}^{i}\right|\right) \leq & E\left(\sup _{t \leq T}\left|Z_{t}^{i n}-\bar{Y}_{t}^{i, n}\right|\right) \\
& +E\left(\sup _{t \leq T}\left|\bar{Y}_{t}^{i, n}-\bar{X}_{t}^{i}\right|\right) \\
\leq & \frac{M_{\varepsilon_{n}}}{\sqrt{n} L_{\varepsilon_{n}}} \exp \left(\left\|w_{0}\right\|_{1} T L_{\varepsilon_{n}}\right)+E\left(\sup _{t \leq T}\left|\bar{Y}_{t}^{i, n}-\bar{X}_{t}^{i}\right|\right) .
\end{aligned}
$$

But

$$
\begin{aligned}
\left|\bar{Y}_{t}^{i, n}-\bar{X}_{t}^{i}\right| \leq & \int_{0}^{t}\left|K_{\varepsilon_{n}} * \tilde{p}_{s}^{n}\left(\bar{Y}_{s}^{i, n}\right)-K_{\varepsilon_{n}} * \tilde{p}_{s}^{n}\left(\bar{X}_{s}^{i}\right)\right| d s \\
& +\int_{0}^{t}\left|K_{\varepsilon_{n}} * \tilde{p}_{s}^{n}\left(\bar{X}_{s}^{i}\right)-K * w\left(\bar{X}_{s}^{i}\right)\right| d s .
\end{aligned}
$$


The second term on the right-hand side is controlled due to Corollary 3.6. It remains to study the first term on the right-hand side, which we do following [7], Lemma 3.1 and Theorem 3.1. It is proved that, for $x$ and $z$ in $\mathbb{R}^{2}$,

$$
\left|K_{\varepsilon_{n}} * \tilde{p}_{s}^{n}(x)-K_{\varepsilon_{n}} * \tilde{p}_{s}^{n}(z)\right| \leq C_{0}\left(\left\|w_{0}\right\|_{1}+\left\|w_{0}\right\|_{\infty}\right) \phi(x, z),
$$

where

$$
\phi(x, z)=\tilde{\phi}(|x-z|),
$$

and $\tilde{\phi}(r)=r(1-\ln r)$ if $0<r<1$ and $\tilde{\phi}(r)=1$ if $r \geq 1$. Let us remark that the function $\tilde{\phi}$ is nondecreasing and concave.

Then, by noting $C=C_{0}\left(\left\|w_{0}\right\|_{1}+\left\|w_{0}\right\|_{\infty}\right)$, one deduces that

$$
\left|\bar{Y}_{t}^{i, n}-\bar{X}_{t}^{i}\right| \leq A_{T} \varepsilon_{n}+C \int_{0}^{t} \phi\left(\bar{Y}_{s}^{i, n}, \bar{X}_{s}^{i}\right) d s .
$$

Then

$$
\begin{aligned}
& E\left(\sup _{u \leq t}\left|\bar{Y}_{u}^{i, n}-\bar{X}_{u}^{i}\right|\right) \\
& \quad \leq A_{T} \varepsilon_{n}+C \int_{0}^{t} E\left(\sup _{u \leq s} \phi\left(\bar{Y}_{u}^{i, n}, \bar{X}_{u}^{i}\right)\right) d s \\
& \quad \leq A_{T} \varepsilon_{n}+C \int_{0}^{t} E\left(\sup _{u \leq s} \phi\left(\bar{Y}_{u}^{i, n}, \bar{X}_{u}^{i}\right)\right) d s \\
& \quad \leq A_{T} \varepsilon_{n}+C \int_{0}^{t} E\left(\tilde{\phi}\left(\sup _{u \leq s}\left|\bar{Y}_{u}^{i, n}-\bar{X}_{u}^{i}\right|\right)\right) d s \text { since } \tilde{\phi} \text { is nondecreasing } \\
& \quad \leq A_{T} \varepsilon_{n}+C \int_{0}^{t} \tilde{\phi}\left(E\left(\sup _{u \leq s}\left|\bar{Y}_{u}^{i, n}, \bar{X}_{u}^{i}\right|\right)\right) d s \quad \text { by concavity of } \tilde{\phi} .
\end{aligned}
$$

Let us denote by $H(t)=E\left(\sup _{u \leq t}\left|\bar{Y}_{u}^{i, n}-\bar{X}_{u}^{i}\right|\right)$. Then, by the previous computations,

$$
H(t) \leq A_{T} \varepsilon_{n}+C \int_{0}^{t} \tilde{\phi}(H(s)) d s .
$$

As in [7], we introduce the solution $h\left(x_{0}, t\right)$ of the equation

$$
z^{\prime}(t)=C \tilde{\phi}(z(t)), \quad z(0)=x_{0}>0 .
$$

Then, if $x_{0}<1$ and if $t_{0}=\inf \left\{t, h\left(x_{0}, t\right)>1\right\}$,

$$
\begin{aligned}
h\left(x_{0}, t\right) & =x_{0}^{\exp (-C t)} \exp \left(1-e^{-C t}\right) \quad \text { if } h\left(x_{0}, t\right)<1, t<t_{0} \\
& =1+C\left(t-t_{0}\right) \quad \text { if } h\left(x_{0}, t\right) \geq 1, t \geq t_{0}
\end{aligned}
$$

and if $x_{0} \geq 1, h\left(x_{0}, t\right)=x_{0}+C t$. Hence, by (3.18), we have

$$
H(t) \leq h\left(A_{T} \varepsilon_{n}, t\right) .
$$


But since $\varepsilon_{n}$ tends to 0 as $n$ tends to $\infty$, for $n$ sufficiently large, we deduce that

$$
H(t) \leq\left(A_{T} \varepsilon_{n}\right)^{\exp (-C t)} \exp \left(1-e^{-C t}\right)
$$

and, finally,

$$
E\left(\sup _{u \leq T}\left|\bar{Y}_{u}^{i, n}-\bar{X}_{u}^{i}\right|\right) \leq\left(A_{T} \varepsilon_{n}\right)^{\exp (-C T)} \exp \left(1-e^{-C T}\right)
$$

for $n$ sufficiently large.

Now, by (3.17), and (3.19), we finally deduce that

$$
\begin{aligned}
& E\left(\sup _{t \leq T}\left|Z_{t}^{i n}-\bar{X}_{t}^{i}\right|\right) \\
& \quad \leq \frac{M_{\varepsilon_{n}}}{\sqrt{n} L_{\varepsilon_{n}}} \exp \left(\left\|w_{0}\right\|_{1} T L_{\varepsilon_{n}}\right)+\left(A_{T} \varepsilon_{n}\right)^{\exp (-C T)} \exp \left(1-e^{-C T}\right),
\end{aligned}
$$

where $C=C_{0}\left(\left\|w_{0}\right\|_{1}+\left\|w_{0}\right\|_{\infty}\right)$ and then tends to 0 when $n$ tends to $\infty$.

\section{REFERENCES}

[1] CHORIN, A. J. (1994). Vorticity and Turbulence. Springer, New York.

[2] Giga, Y., Miyakawa, T. and Osada, H. (1988). Two-dimensional Navier-Stokes flow with measures as initial vorticity. Arch. Rational Mech. Anal. 104 223-250.

[3] JouRDAIN, B. (2000). Diffusion processes associated with nonlinear evolutions equations for signed measures. MCAP. To appear.

[4] Jourdain, B. and MÉLÉARD, S. (1998). Propagation of chaos and fluctuations for a moderate model with smooth initial data. Ann. Inst. H. Poincaré Probab. Statist. 34 475-492.

[5] Karatzas, I. and Shreve, S. E. (1991). Brownian Motion and Stochastic Calculus, 2nd ed. Springer, New York.

[6] Lewandowski, R. (1997). Analyse mathématique et océanographie. Recherches en mathématiques appliquées. Masson, Paris.

[7] Marchioro, C. and Pulvirenti, M. (1982). Hydrodynamics in two dimensions and vortex theory. Comm. Math. Phys. 84 483-503.

[8] MÉlÉARD, S. and RoELly-CopPoletta, S. (1987). A propagation of chaos result for a system of particles with moderate interaction. Stochastic Process. Appl. 26 317-332.

[9] Meyer, P. A. (1966). Probabilitiés et potentiel. Hermann, Paris.

[10] OsAdA, H. (1987). Propagation of chaos for the two dimensional Navier-Stokes equations. In Probabilistic Methods in Mathematical Physics (K. Itô and N. Ikeda, eds.) 303-334. Kinokuniya, Tokyo.

[11] Stroock. D. W. and Varadhan, S. R. S. (1979). Multidimensional Diffusion Processes. Springer, New York.

[12] Sznitman, A. S. (1991). Topics in propagation of chaos. Lecture Notes in Math. 1464. Springer, New York.

UNIVERSITÉ PARIS 10

MODAL'X (JE 421)

200 AVENUE DE LA RÉPUBLIQUE 92000 NANTERRE

FRANCE

E-MAIL: sylm@ccr.jussieu.fr 